\title{
Archéopages
}

Archéopages Archéologie et société

45 | 2017

De la terre au pot

\section{De la signature technique à la signature ethnique}

Les productions céramiques locales des $\mathrm{II}^{\mathrm{e}}-\mathrm{I}^{\mathrm{er}}$ siècles avant notre ère à I Palazzi

From technical signature to ethnic signature. Local ceramic productions from the 2nd-1st centuries BC to I Palazzi

De la firma técnica a la firma étnica. Las producciones cerámicas locales en I Palazzi durante los siglos II y I antes de nuestra era

\section{Kewin Peche-Quilichini}

\section{(2) OpenEdition} Journals

Édition électronique

URL : https://journals.openedition.org/archeopages/2644

DOI : $10.4000 /$ archeopages. 2644

ISSN : 2269-9872

Éditeur

INRAP - Institut national de recherches archéologiques préventives

Édition imprimée

Date de publication : 1 mars 2018

Pagination : 20-31

ISSN : 1622-8545

Référence électronique

Kewin Peche-Quilichini, « De la signature technique à la signature ethnique », Archéopages [En ligne], 45 | 2017, mis en ligne le 01 janvier 2020, consulté le 03 juin 2021. URL : http://

journals.openedition.org/archeopages/2644; DOI : https://doi.org/10.4000/archeopages.2644 


\section{Expérimentation de chaînes opératoires}

Kewrin Peche-Quilichini Inrap, UMR 5140, «Archéologie des Sociétés Méditerranéennes "

L'archéologie expérimentale constitue une voie encore peu utilisée dans le domaine des études céramologiques ; on lui préfère souvent l'apport documentaire fourni par les observations réalisées en contexte archéo-ethnographique. On considère néanmoins généralement que la reconstitution hypothétique des gestes des potiers à partir des résultats de l'analyse technologique peut constituer un complément efficace dans le cadre de restitution des chaînes opératoires, surtout pour les productions façonnées manuellement (Martineau, 2001, 2010). Dans cette optique, une expérimentation a concerné différents protocoles de traitement des surfaces des récipients corses de la fin du premier millénaire avant notre ère.

La caractérisation fine (profondeur, ampleur, largeur, direction, sens, position sur le vase, organisation par rapport au profil du récipient superpositions, etc.) des macrotraces linéaires parallèles présentes sur les parois des poteries corses de la fin de l'âge du Fer nous a conduit à nous interroger sur le type d'outil utilisé pour les réaliser. Dès les premières observations, les archéologues insulaires ont en effet collégialement admis l'idée de l'usage systématique d'un peigne (ou d'un autre outil pectiniforme) dans ce cadre. Néanmoins, ce postulat n'avait jamais été testé dans un cadre expérimental. On présente donc ici une approche de ce type afin de remédier à cette lacune et tester différents scenarii techniques. Une centaine de carreaux de $7 \times 7 \times 1 \mathrm{~cm}$ ont été modelés et lissés au galet pour servir de supports. Ils présentent une surface aplanie et ne correspondent donc pas tout à fait à une paroi légèrement convexe (paroi externe) ou concave (paroi interne) de récipient. Nous avons utilisé un mélange homogène de $50 \%$ d'argile industrielle et de $50 \%$ d'argile collectée sur le gîte Benista 2 (commune de Grosseto-Prugna, Corse-du-Sud). Cette matrice a ensuite été dégraissée au moyen d'arènes détritiques calibrées sous $4 \mathrm{~mm}$. Les tests ont été réalisés sur deux types de carreaux : carreau " consistance cuir » ( 2 heures de séchage ; le traitement superficiel se faisant après l'achèvement du façonnage, il s'agissait d'estimer un délai un peu supérieur à celui estimé entre les deux opérations) et carreau « consistance cuir » à surface réhumidifiée " (cette opération pouvait potentiellement faciliter les traitements superficiels après un délai de séchage inhérent au temps nécessaire pour le façonnage). Dans le but de confronter les traces obtenues aux macrotraces archéologiques, trois items de surfaçage ont été expérimentés (selon des directions rectilignes et croisées) : le lissage au doigt, le brossage (au moyen d'un pinceau à poil animal) et le peignage (au moyen d'un peigne moderne à dents de bois). Les résultats de l'expérimentation invalident les hypothèses du lissage digital et du brossage. Fn effet, les traces laissées par le doigt diffèrent des vestiges archéologiques surtout par leur superficialité alors que celles du pinceau sont trop serrées et relativement peu profondes. Le brossage provoque en outre un effacement couvrant de la plage lissée dont l'aspect s'apparente à celui d'un guillochage. Pour le lissage au doigt comme pour le brossage, le croisement de la direction du geste ne provoque aucune réaction particulière, si ce n'est l'effacement partiel des stigmates du premier traitement superficiel. Le peignage laisse quant à lui des traces tout à fait comparables à celles des échantillons archéologiques. L'opération de ce protocole sur pâte sèche entraîne un arrachage de la couche superficielle d'argile. En revanche, le peignage sur pâte réhumidifiée est pleinement satisfaisant, à condition que la paroi soit lisse et plate (ou à cintre accentué) et garantisse un degré de couvrement optimal. Le peignage orthogonal entraîne quelques décollements de plages d'argile qui renvoient à des observations faites sur une minorité de tessons de l'âge du Fer.

L'expérimentation permet de conclure à l'usage d'un peigne à dents d'égale longueur sur paroi lissée et humide, probablement utilisé de façon immédiatement postérieure à une réhumidification au doigt (re-lissage ?) des parois, ce qui semble de plus confirmé par quelques impacts accidentels de dents de peigne le plus souvent imprimés au moment de retirer l'outil. Malgré ces observations, l'aspect des peignes, la longueur et le nombre des dents ne peuvent à ce jour être déterminés.

Références bibliographiques

MARTINEAU R., 2001, « La fabrication des poteries du groupe de Clairvaux ancien (Jura, France) entre 3025 et 2980 avant J.-C. Expérimentation et analyses du façonnage et traitements de surface », in Bourguignon L., Ortega I., Frère-Sautot M.-C. (dir.), Préhistoire et approche expérimentale, Préhistoires, 5, p. 173-186.

MARTINeAu R., 2010, « Brunissage, polissage et degrés de séchage. Un référentiel expérimental», in Approches de la chaîne opératoire de la céramique Le façonnage, Les Nouvelles de l'Archéologie, 119, p. 13-19. 
Séchage : $2 \mathrm{~h}$

Direction unique

Séchage : $2 \mathrm{~h}$

Pâte réhumidifiée

Direction unique

Séchage : $2 \mathrm{~h}$

Direction croisée

Séchage : $2 \mathrm{~h}$

Pâte réhumidifiée

Direction croisée
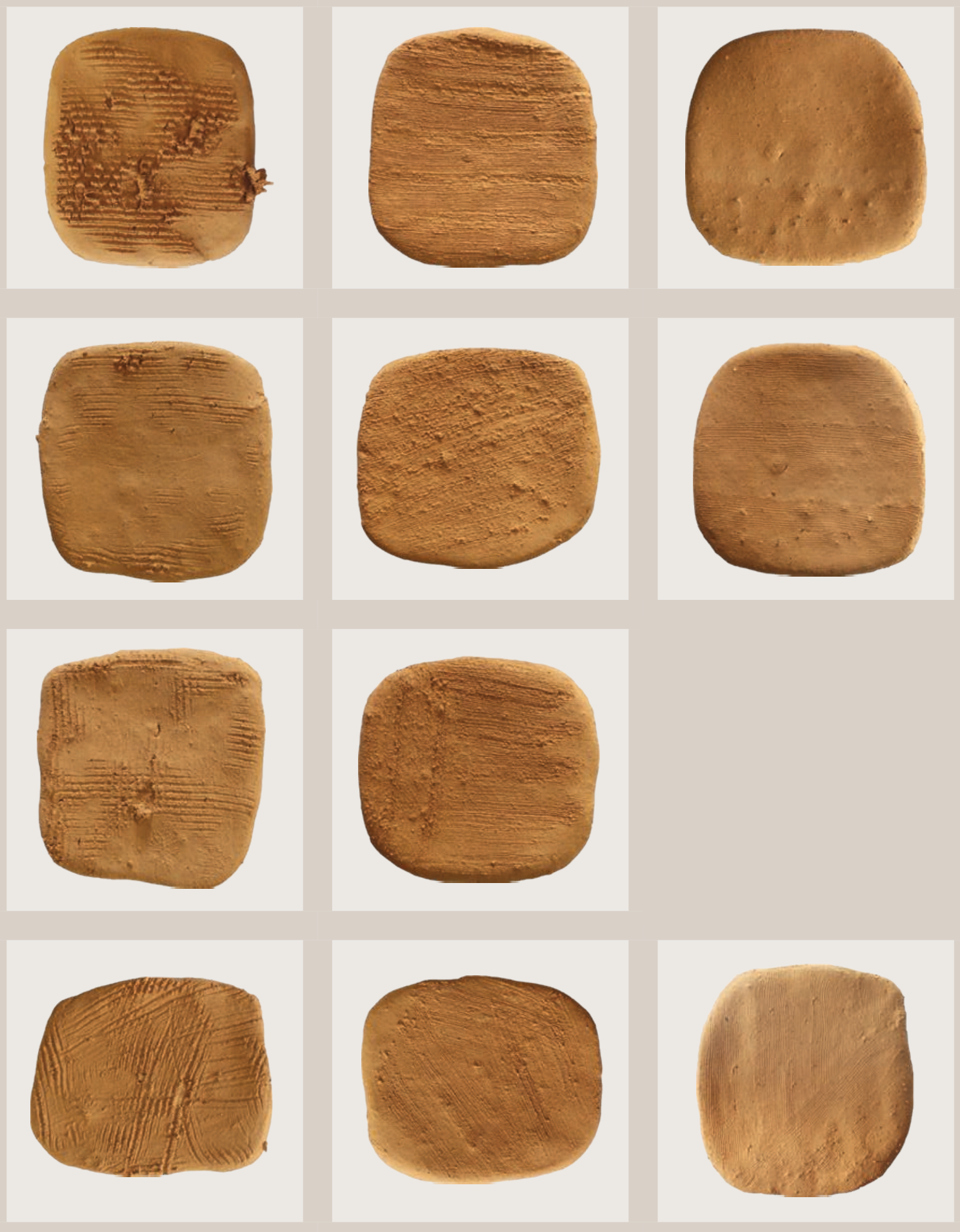

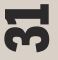

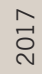

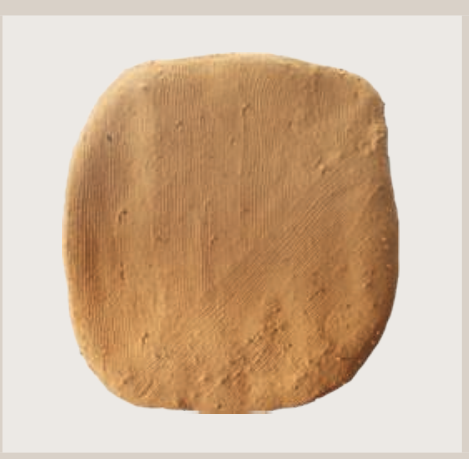

Résultats de

l'expérimentation visant

à restituer les macrotraces

laissées par un lissage

au doigt, un brossage et

un peignage sur pâte après

2 heures de séchage

et sur pâte réhumidifiée 